\title{
Recent Advancements and Applications in the Analysis of Traditional Chinese Medicines
}

Author

Affiliation
Markus Ganzera

Institute of Pharmacy - Pharmacognosy, University of Innsbruck, Innsbruck, Austria

\author{
Key words \\ - traditional Chinese medicine \\ - TCM \\ - analysis \\ - quality control
}

received January 21, 2009 revised March 11, 2009 accepted March 24, 2009

Bibliography

DOI $10.1055 / \mathrm{s}-0029-1185686$

Published online May 8, 2009

Planta Med 2009; 75: 776-783

(c) Georg Thieme Verlag KG

Stuttgart · New York .

ISSN 0032-0943

\section{Correspondence}

Priv. Doz. Dr. Markus Ganzera

Institute of Pharmacy -

Pharmacognosy

University of Innsbruck

Innrain 52

6020 Innsbruck

Austria

Phone: + 435125075307

Fax: + 435125072939

markus.ganzera@uibk.ac.at

\section{Introduction} $\nabla$

Traditional Chinese medicine (TCM) is based on a totally different concept than Western medicine. It does not only consider single factors being responsible for a certain disease but the unbalanced status of the whole organism ("holism") and its interaction with the environment. Relevant to

\section{A}

One of the general hurdles limiting the application and development of traditional Chinese medicines (TCM) is their occasional poor or inconsistent quality. Being multi-component mixtures, however, assuring the quality of such preparations is a challenging task. In recent years significant analytical advancements and innovations were made in relation to this particular problem among others. This review summarizes respective reports within the last five years, and reflects the multitude of current methods the analyst can choose from. Besides established techniques, such as HPLC or CE, analytical methods also include more "exotic" ones like HSCCC, CEC, biologically based assays, and the use of IR spectroscopy. Fingerprinting techniques, which are especially popular for analyzing TCM remedies, are also mentioned. Selected examples for currently observable trends will be presented and briefly discussed so that the current status of TCM analysis is clearly visualized.

\section{Abbrevations \\ $\checkmark$ \\ ASE: accelerated solvent extraction \\ CE: capillary electrophoresis \\ CEC: capillary electrochromatography \\ ELSD: evaporative light scattering detection \\ FSCE: free solution capillary electrophoresis}

FT-IR: $\quad$ Fourier transform infrared

HS-SDME: headspace single-droplet microextraction

HS-SPME: headspace solid-phase microextraction

HILIC: hydrophilic interaction liquid chromatography

HSCCC: high speed counter current chromatography

ICP-AES: inductively coupled plasma atomic absorption emission spectroscopy

LOD: limit of detection

MAR: macroporous adsorptive resins

MEEKC: micro emulsion electrokinetic chromatography

MEKC: micellar elecktrokinetic chromatography

MIP: $\quad$ molecular imprinted polymer

MIR: mid infrared

NACE: non-aqueous capillary electrophoresis

NIR: near infrared

PCR: polymerase chain reaction

RRLC: rapid resolution liquid chromatography

RSLC: rapid separation liquid chromatography

TCM: traditional Chinese medicine

TOF: time of flight

UPLC: $\quad$ ultra performance liquid chromatography this are seasonal and geographical variations. In brief, the human body is centered around five "zang" organs (heart, liver, spleen, lung and kidney), which are connected by meridians interacting with the exterior by sensory organs and orifices. Viscera, though different in function, interact with each other. Thus, diseases will have an impact not on one but on several organs, and maybe 


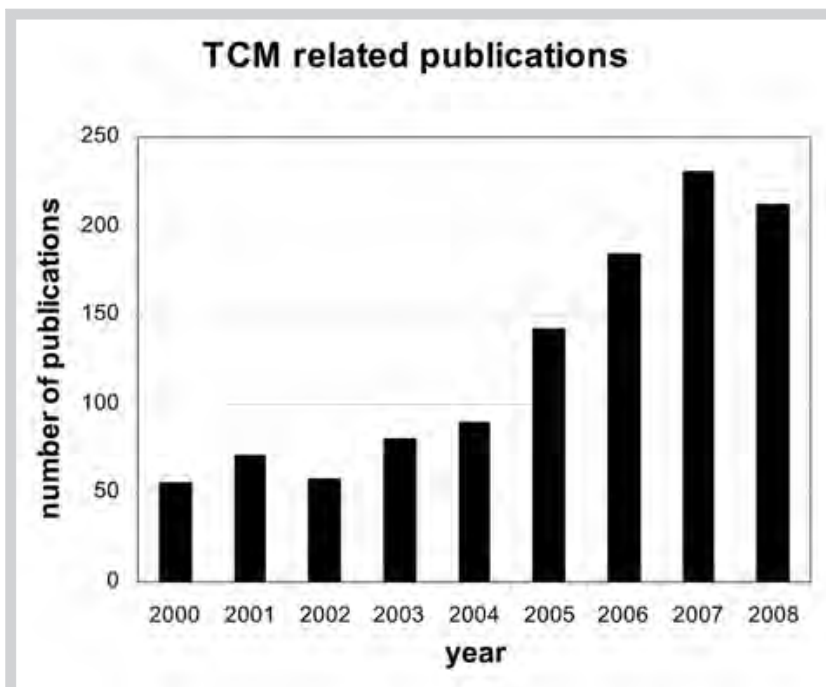

Fig. 1 Number of SciFinder database hits when using the search terms "TCM" and "analysis".

the whole body. Therapy starts with differentiation of the syndrome (e.g., based on particular symptoms, patient counselling, pulse taking and palpation), followed by an appropriate treatment. The latter includes the use of mostly complex herbal preparations, acupuncture or moxibustion (stimulation of acupuncture points by heat treatment). Due to the holistic approach of TCM the same disease might show different syndromes, therefore requiring a different form of treatment [1-3].

Alternative medicines including TCM, Ayurveda and others are increasingly becoming popular in the Western world. They are trendy and considered less harmful because of their "natural" ingredients. This is definitely not true as indicated by toxic side effects of particular herbs or adulterations [4-6]. The situation is further complicated by the fact that some of these products are distributed via the internet, without any assurance of correct plant identification or quality. Regulatory institutions and the scientific community are aware of this problem, and work on the development of analytical methods for quality control of respective ingredients and preparations ( $\bullet$ Fig. 1 shows SciFinder hits for the combined search terms "TCM" and "analysis" listed by publication year). The technique by far most commonly applied for this purpose is HPLC (in approx. 60\% of all relevant 2008 publications utilized), followed by GC and CE. It is noteworthy that most of the reports describe the analysis of single herbal components in TCM plants and not in the entire complex formulation.

The goal of this review is to summarize recent developments in the analysis of TCM preparations, whereby the format of a minireview does not allow a comprehensive evaluation of the whole topic. However, an informative and excellent review has recently been published by Liu et al. [7]. Therefore, in this manuscript routine techniques such as the above-mentioned ones will only briefly be addressed, focusing on more uncommon and innovative approaches instead. The observed period includes the last five years (2004 to 2008), considering publications in English only. Separation techniques are also involved in sample preparation prior to actual analysis; recent advancements in this area (e.g., MAR, MIP) have been addressed elsewhere already [7-9].

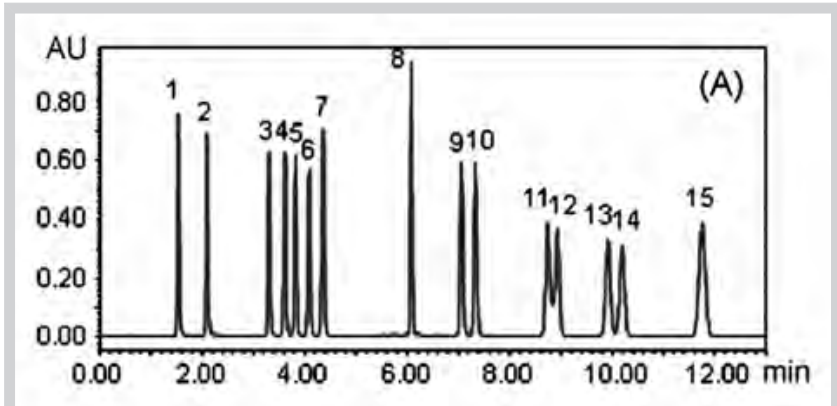

Fig. 2 Separation of 15 flavonoids present in different Epimedium species by UPLC [(1) hexandraside E; (2) kaempferol-3-O-rhamnoside; (3) hexandraside F; (4) pimedin A; (5) epimedin B; (6) epimedin C; (7) icariin; (8) epimedoside C; (9) baohuoside II; (10) caohuoside C; (11) baohuoside VII; (12) sagittatoside A; (13) sagittatoside B; (14) 2-O-rhamnosylicariside II; (15) baohuoside I]. Reproduced with permission from [16].

\section{Chromatographic Methods \\ $\nabla$}

High performance liquid chromatography (HPLC)

Numerous applications of LC in combination with various detectors can be found in the literature for the assessment of TCM. They include, to name a few, analysis of " $\mathrm{Qi}-$ Shen-Yi-Qi preparation" by LC-UV and LC-ELSD [10], determination of flavonoids in Hypericum japonicum by HPLC-ESI-MS [11] and the simultaneous assay of phenolic compounds, alkaloids, saponins and flavonoids in diverse TCM prescriptions using LC-TOF-MS [12].

A comparably new trend is the use of sub- $2 \mu \mathrm{m}$ stationary phases for separation (UPLC, RRLC, RSLC). These materials show dramatically increased separation efficiency at simultaneously reduced separation time, but result in much higher column backpressure. In addition to "fast" detectors with a high sampling rate, special pumps enabling pressures up to 1000 bar are therefore required. Several species of the Panax genus have been investigated by this technique, for example Chan and coworkers studied raw and steamed Panax notoginseng [13]. By using a $1.7 \mu \mathrm{m}$ column material (Waters Aquity C-18; $100 \times 2.1 \mathrm{~mm}$ ) and a mobile phase comprising water and $0.1 \%$ of formic acid in acetonitrile they were able to assign all major ginsenosides (23 compounds) in less than $8 \mathrm{~min}$. Respective compounds were identified based on reference standards and TOF-MS data. Similar applications on the abovementioned species as well as $P$. ginseng, $P$. japonicus and $P$. quinquefolium were described in two other reports [14,15]. According to the Chinese pharmacopoeia "Yin-Yang-Huo", several different Epimedium species are comprised under this name, can be used to lower blood pressure and to treat diabetes. In order to differentiate these species based on their flavonoid pattern Chen et al. have developed a respective UPLC assay ( $\odot$ Fig. 2) [16]. Plant material was first extracted with $70 \%$ ethanol using ASE conditions $\left(120^{\circ} \mathrm{C}\right.$ at $\left.1500 \mathrm{psi}\right)$, and then separated on a 5-cm long Aquity BEH C-18 column. In only $13 \mathrm{~min}$ a total of 15 flavonoid glycosides could be separated in herbal samples and quantified by UV detection with excellent sensitivity (LOD from 0.05 to $0.13 \mathrm{ng}$ ) and precision (RSD for intraday precision $\leq 3.1 \%$ ). Technically similar applications are those by Jin et al. on the analysis of quinochalcones in Carthamus tinctorius [17] and three marker compounds in Eleutherococcus senticosus [18].

Whenever very polar compounds are to be analyzed by reversed phase chromatography insufficient retention is a commonly observed problem. It can be overcome by the use of ion-pairing re- 
agents or novel HILIC materials. The latter show selectivity similar to normal phase, but aqueous mobile phases (water has the highest solvent strength on this phase) can be employed [19]. An interesting application of this technique on TCM products was reported by Wang and coworkers in 2008 [20]. They used column-switching (column 1: Atlantis HILIC silica, mobile phase: acetonitrile and $100 \mathrm{mM}$ aqueous ammonium acetate; column 2: Hypersil ODS 2, mobile phase: $0.1 \%$ formic acid in water and acetonitrile) to separate polar (column 1) as well as non-polar (column 2) constituents in Lonicera japonica. The system comprised two sets of LC modules, which were hyphenated through an interface with an electronically controlled valve. Through this interface transfer and separation of those analytes not retained on column 1 was achieved on column 2; detection of the individual analytes was carried out at $254 \mathrm{~nm}$.

Another rather uncommon approach for studying the composition of herbal material and TCM preparations in particular is the use of two-dimensional HPLC. Like the above-described system two columns are required, but after separation on the first column small portions of the eluent are trapped (online or offline), and then assayed on the second one. Both columns need to have different separation mechanisms, and the separation of the second dimension must not be less than the resolution of the first column [21]. If these requirements are fulfilled, total component analysis with high resolution is possible. Hu et al. studied two important TCM plants, Ligusticum chuanxiong and Angelica sinensis, by this technique [22]. Methanolic extracts were first separated on $\mathrm{CN}$ material $(5 \mu \mathrm{m}$ Kromasil $\mathrm{CN} ; 150 \times 4.6 \mathrm{~mm}$, flow rate $0.13 \mathrm{~mL} / \mathrm{min}$ ), the eluent trapped online in a loop, and rapidly analyzed on a Chromolith monolithic column from Merck (50× $4.6 \mathrm{~mm}$, flow rate $3.0 \mathrm{~mL} / \mathrm{min}$ ). For both separations, gradient elution with aqueous acidic acetonitrile was used, and detection was achieved by UV and MS. After visualization of the results in a 3-D plot more than 100 compounds could be separated and identified in both plants, respectively. The offline coupling of two columns (separation and fractionation on the first, analysis of the fractions on the second column, and combination of results) has been described as well. By combining HILIC (in-house made click $\beta-C D$ material) and reversed phase material (Inertsil ODS) the composition of Carthamus tinctorius was studied [23]. Ma et al. utilized size-exclusion chromatography (Toyopearl HW-40S) and RP-material (Hypersil ODS-C18) to investigate "Qingkailing", a TCM preparation composed of eight herbal ingredients; 54 components could be separated, and seven identified based on MS data [24].

\section{Gas chromatography (GC)}

Gas chromatography has always been and most likely will be the method of choice for analyzing essential oils. As such, numerous GC applications on TCM relevant herbs can be found in the recent literature, many of them utilizing MS for detection. In respect to separation and detection little new is described compared to earlier reports. What seems to be more interesting are advancements regarding sample preparation. For example, a procedure commonly applied is headspace solid-phase microextraction (HS-SPME). In a first step volatile constituents are transferred from the matrix to the headspace phase by thermal or microwave heating. They are adsorbed on suitable SPME materials (fibers coated with stationary phases such as polysiloxanes or divinylbenzene) and then desorbed in the GC apparatus at increased temperature again. Once a suitable SPME material has been selected, the simple, fast and solvent-free extraction and enrich- ment of particular compounds is possible. Successful applications of this technique on TCM plants include the analysis of ligustilides in Ligusticum chuanxiong [25], the essential oil of Chrysanthemum [26,27] or that of Artemisia argyi flowers [28].

A modification of the aforementioned procedure is HS-SDME, whereby SD stands for single droplet. Here, the analytes are extracted using a single microdrop (1-5 $\mu \mathrm{L})$ of solvent such as octanol or benzyl alcohol, which is placed in the headspace phase through the tip of a microsyringe. After an appropriate time at a suitable temperature, the droplet is withdrawn into the syringe, and analyzed by MS. Deng and coworkers determined the content of paeonol in two TCM plants, Paeonia suffruticosa and Cynanchum paniculatum, by this approach [29]. They first prepared an aqueous extract by microwave-assisted extraction, and then extracted the target compound in a single $1 \mu \mathrm{L}$ drop of octanol. Exposure time was $4 \mathrm{~min}$ at a temperature of $50{ }^{\circ} \mathrm{C}$ and stirring rate of $700 \mathrm{rpm}$. With RSD values of less than $10.5 \%$ for repeated analysis and recoveries for paeonol of over $88 \%$ the authors considered this method to be well suited for routine analysis. In a similar fashion the analysis of $Z$-ligustilide, one of the biologically active constituents in Angelica sinensis essential oil, in rabbit plasma has been described [30].

\section{High speed counter current chromatography (HSCCC)}

High speed counter current chromatography is a support-free liquid-liquid partition chromatographic technique invented by Ito and Bowman in the early 1970s [31]. By eliminating the use of a solid stationary phase loss of constituents due to irreversible adsorption is avoided. Instead, two immiscible solvents or mixtures thereof are employed, with the stationary phase being retained in a coil of flexible length by centrifugal forces and the mobile phase driven through the system by external pumping. The compounds of interest are separated based on their different partition coefficients. More details regarding separation theory and instrumental set-up can be found in a very informative recent review by Ito [32].

Because of scale-up options and obvious advantages compared to column chromatography (see above) this technique is mainly used for preparative purposes rather than analytical applications. Especially for the isolation of constituents from TCM remedies HSCCC has gained some popularity, and Liu and coworkers have already compiled these methods in their review [7]. As can be seen, mainly the isolation of flavonoids (e.g., in Epimedium koreanum or Glyzyrrhiza inflata), alkaloids (e.g., Evodia rutaecarpa, Huperzia serrata) and coumarins (e.g., Cnidium monnieri) has been described, and reported purities of usually more than $95 \%$ impressively demonstrate the potential of this approach.

Analytical reports utilizing HSCCC are scarce. One example with TCM relevance is the study published by Wang et al. [33]. They present three successful applications (Arctium lappa, Magnolia officinalis and Psoralea corylitolia), concluding that they consider HSCCC suitable for controlling the quality of herbal raw materials. However, only qualitative results are presented, but particularly the separation of biphenyls (magnolol and honokiol) in a crude ethanolic extract of $M$. officinalis seems promising ( $\odot$ Fig. 3 ). The solvent system comprised a mixture of $n$-hexane, ethyl acetate, methanol and water, in the ratio of $1: 0.4: 1: 0.4$. Separations were performed in an analytical separation column of $36 \mathrm{~mL}$ volume, at a revolution speed of $1800 \mathrm{rpm}$, and flow rate of $2.0 \mathrm{~mL} /$ min. For detection a UV-VIS photometer set to $254 \mathrm{~nm}$ was utilized. HSCCC has also been employed for fingerprint analysis of TCM preparations, particularly those containing Salvia miltiorrhi- 


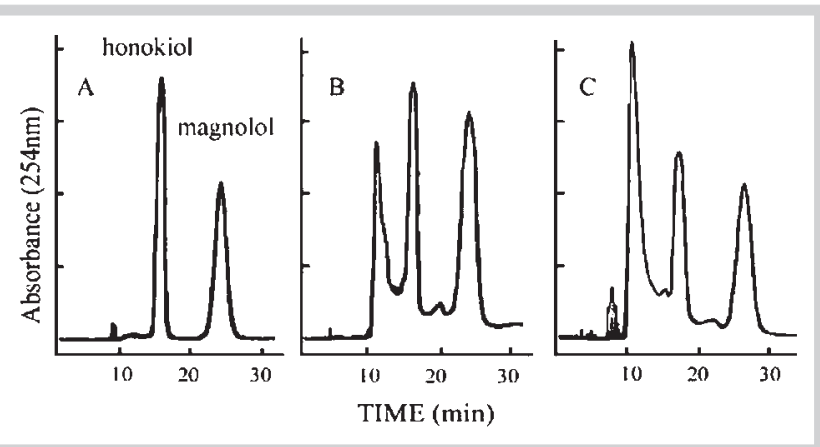

Fig. 3 HSCCC separation of the biphenyls honokiol and magnolol (A: standard mixture; $\mathbf{B}$ and $\mathbf{C}$ : two different Magnolia officinalis crude extracts); detection at $254 \mathrm{~nm}$. Reproduced with permission from [33].

$z a$, "Tan-Shen" in Chinese; for further details see the respective section of this review.

\section{Electrophoretic Methods \\ $\nabla$}

\section{Capillary electrophoresis (CE)}

Capillary electrophoresis offers many unique features and modes that explain why this technique is increasingly used for natural products analysis. Separation is commonly achieved by the different mobility of charged analytes in an electric field, either in aqueous (FSCE) or non-aqueous buffers (NACE). If non-charged molecules are to be resolved, electrolytes with charged pseudostationary phases formed by micelles (MEKC) or solvent droplets (MEEKC) can be employed. These techniques are especially popular among Chinese researchers. Therefore, listing of all TCM related applications would go far beyond the scope of this minireview, especially as a recent review by Ganzera has covered this topic in detail [34].

\section{Capillary electrochromatography (CEC)}

This technique utilizes the same driving force as capillary electrophoresis, which is the electro-osmotic flow, but separation is achieved because of chromatographic interaction. Therefore CEC can be considered as the true hybrid of HPLC and CE, combining the benefits of both, i.e., high separation efficiency and low sample/solvent consumption. Stationary phase (e.g., regular RP-18 material with small particle size) is either filled in silica capillaries with an internal diameter of around $100 \mu \mathrm{m}$, or it is in situ polymerized in the capillaries. The latter are called monolithic columns, whereby silica and synthetic polymer (e.g., methacrylate, divinylbenzene) based monoliths have been described. Since CEC is a comparatively new development, only a few applications with reference to TCM can be found in the literature.

The use of packed CEC capillaries is described for the analysis of five coumarins (xanthotoxol, osthenol, imperatorin, oxypeucedanin hydrate and byankangelicin) in Angelica dahurica ("Bai-Zi" in Chinese) [35], the determination of nucleosides in Cordyceps sinensis [36], and the determination of flavonoids in Herba Epimedii ("Yin-Hang-Huo") [37]. In all of these reports the same stationary phase was selected ( $3 \mu \mathrm{m}$ Hypersil C-18 material), but the mobile phases employed were slightly different. For example, Chen et al. [37] achieved optimum results with a mixture of $20 \mathrm{mM}$ phosphate buffer ( $\mathrm{pH} 4.0$ ) and acetonitrile in the ratio of

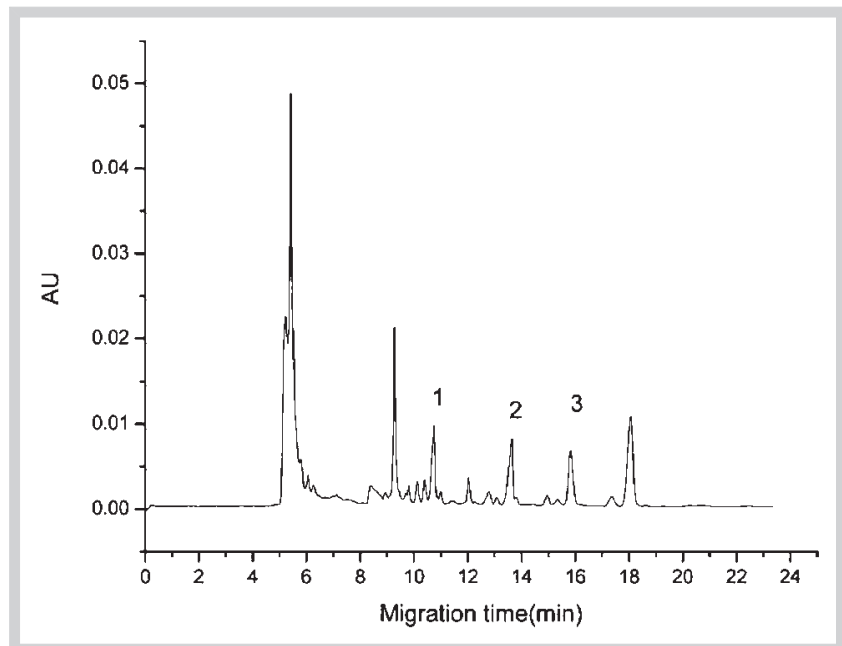

Fig. 4 Determination of quaternary alkaloids in Coptis chinensis by monolithic CEC [(1) palmatine, (2) berberine, (3) jatrorrhizine]. Reproduced with permission from [41].

$7: 3$. The applied voltage and temperature were $30 \mathrm{kV}$ and $25^{\circ} \mathrm{C}$, respectively. With these settings an excellent separation of eight flavonoids was accomplished in less than $20 \mathrm{~min}$, also enabling their quantitative determination in crude plant material (LOD of individual flavonoids between 4.1 to $8.6 \mu \mathrm{g} / \mathrm{mL}$ ).

Nearly all of the few reports regarding the application of monolithic CEC materials describe the use of polymeric materials. This is explainable by the fact that they, even containing no secondary structure (mesopores), are easier to prepare and more pH-stable than silica-based ones [38]. For example, Lü elaborated on the quantitative determination of structurally related anthraquinones in rhubarb on a stationary phase directly polymerized in $100 \mu \mathrm{m}$ I.D. capillaries [39]. A mixture of methacrylic acid and three methacrylates was prepared in 1-propanol and 1,4-butanediol, and polymerization was thermally induced by azobisisobutyronitrile. For the actual separation of plant extracts a $10 \mathrm{mM}$ phosphate buffer pH 6.2 containing 65\% acetonitrile was used. Zhang and coworkers reported on the analysis of flavonoids in Adinandra nitida leaves on similar monoliths [40]. This plant is commonly utilized in TCM because of its antitumor properties. Since isocratic conditions did not allow the separation of all compounds of interest, the amount of acetonitrile in a phosphate buffer was gradually increased during analysis. Quantitative results for apigenin and epicatechin in A. nitida samples were presented as well.

The composition of the polymerization mixture is quite flexible, to the effect that stationary phases with diverse chromatographic properties can be prepared rather easily. One option in this respect is the introduction of charged functionalities, thereby producing CEC materials with ion-exchange character. Accordingly, the analysis of alkaloids on cation-exchange monoliths has been attempted for those in Coptis chinensis ( $\odot$ Fig. 4, [41]) and opium [42]. Although the successful application on plant extracts has been demonstrated in these studies, they are mainly focused on mechanistic investigations, and detailed quantitative results are lacking. 
Table 1 Recent examples of fingerprint analysis of TCM plants and preparations.

\begin{tabular}{|c|c|c|c|c|}
\hline & Sample & Technique & Conditions & Ref. \\
\hline \multirow[t]{12}{*}{ Individual plants } & Angelica dahurica & HPLC & Zorbax Extend C-18, methanol, water, $270 \mathrm{~nm}$ & [48] \\
\hline & Angelica sinensis & FT-IR, 2D-IR & Scan range $400-4000 \mathrm{~cm}^{-1}$, resolution $4 \mathrm{~cm}^{-1}$ & [49] \\
\hline & Atractylis chinensis & HPLC, ICP-AES & HPLC: Zorbax Eclipse-XDB C18 column, methanol, $0.1 \%$ phosphoric acid, $320 \mathrm{~nm}$ & [50] \\
\hline & Caulophyllum robustum & HPLC, GC & $\begin{array}{l}\text { HPLC: Zorbax SB-C } 18,0.01 \% \text { phosphoric acid, methanol, acetonitril, } 267 \text { nm; } \\
\text { GC: DB-5MS column, MS detection }\end{array}$ & [51] \\
\hline & Coptis chinensis & UPLC & $\begin{array}{l}\text { Aquity UPLC BEH C }{ }_{18}, 0.5 \% \text { acetic acid in phosphate buffer, acetonitrile, 230, } 265 \\
\text { and } 350 \mathrm{~nm}\end{array}$ & [52] \\
\hline & Epimedium spp. & FT-IR & Scan range $400-4000 \mathrm{~cm}^{-1}$, resolution $4 \mathrm{~cm}^{-1}$ & [53] \\
\hline & Ganoderma lucidum & HPLC & Kromasil C18, $0.1 \%$ acetic acid, acetonitrile, $254 \mathrm{~nm}$ & [54] \\
\hline & Ginkgo biloba & HPLC & CenturySIL C18 BDS, $1 \%$ acetic acid in water and acetonitrile, $265 \mathrm{~nm}$ & [55] \\
\hline & Ligusticum chuanxiong & PCEC, HPLC & $\begin{array}{l}\text { PCEC: Unimicro EP-150-30, } 0.02 \% \text { TFA, methanol, water, }-10 \mathrm{kV}, 280 \mathrm{~nm} \text {; } \\
\text { HPLC: Elite Symmerty C18, } 0.5 \% \text { phosphoric acid, methanol, } 280 \mathrm{~nm}\end{array}$ & [56] \\
\hline & Rehmannia glutinosa & GC & HP-5MS column, MS detection & [57] \\
\hline & Salvia miltiorrhiza & NACE, HSCCC & $\begin{array}{l}\text { NACE: } 125 \mathrm{mM} \text { borax and } 50 \mathrm{mM} \text { sodium deoxycholate in methanol, } 25 \mathrm{kV} \text {, } \\
280 \mathrm{~nm} ; \mathrm{HSCCC}: n \text {-hexane/ethanol/water }=10 / 7 / 3,2 \mathrm{~mL} / \mathrm{min}, 900 \mathrm{rpm}, 280 \mathrm{~nm}\end{array}$ & [58] \\
\hline & Salvia miltiorrhiza & TLC, HSCCC & $\begin{array}{l}\text { TLC: silica } 60 \text { F } 254 \text { plates, light petroleum/ethyl acetate = 8/2, } 280 \text { nm; } \\
\text { HSCCC: same as [ } 56 \text { ] }\end{array}$ & [59] \\
\hline \multirow[t]{2}{*}{ Formulations } & Danning tablets & HPLC & $\begin{array}{l}\text { Zorbax Extend } \mathrm{C}-18,12.6 \mathrm{mM} \text { formate buffer } \mathrm{pH} 5 \text {, acetonitrile, } 346 \mathrm{~nm} \text { and MS } \\
\text { detection }\end{array}$ & [60] \\
\hline & $\begin{array}{l}\text { Longdan-Xiegan } \\
\text { decoction }\end{array}$ & 2D-HPLC & $\begin{array}{l}\text { First column: silica-bonded human serum albumin column, } 10 \mathrm{mM} \text { ammonium } \\
\text { acetate solution, acetonitrile, } 0.1 \mathrm{~mL} / \mathrm{min} \text {; second column: Chromolith speed ROD, } \\
\text { water, acetonitrile, } 3 \mathrm{~mL} / \mathrm{min}, 254 \mathrm{~nm} \text { and MS detection }\end{array}$ & [61] \\
\hline
\end{tabular}

\section{Spectroscopic Methods \\ $\nabla$}

Compared to chromatography, spectroscopic methods show unique features and advantages. They combine speed and nondestructive character with the need for no or only little sample preparation. This might be the reason why NIR spectroscopy is especially popular for purity or identity evaluation in industry. Quantitative determinations are possible as well, but they require external calibration with a "conventional" method (e.g., GC, HPLC) prior to actual analysis [43]. Further methodological details can be found in a respective review article in this issue.

Few reports on quantitative IR applications have been published in connection to TCM. Applications for fingerprinting are summarized in the next section. $\mathrm{Wu}$ and colleagues described the use of MIR and NIR spectroscopy for the quantitative determination of $\alpha$-pinene, methyl salicylate and eugenol in "Honghua oil" [44]. This preparation is composed of several essential oils (wintergreen, turpentine, glove and cassia leaf) and is utilized in the treatment of rheumatism and joint problems. For the compounds of interest, characteristic absorption bands in the IR spectra were assigned, and based on quantitative GC results, the areas of these bands were correlated with the respective quantities. Afterwards, constructed calibration curves showed correlation coefficients of 0.98 and higher, and the predicted quantitative results were in good agreement with GC (prediction bias varied from -0.143 to 0.178 ). Results obtained based on the MIR spectra were shown to be slightly more precise.

Another study related to heavy metals in TCM formulations is also noteworthy. By using thermolysis and atomic absorption spectroscopy Huang et al. investigated mercury levels in 11 products and two single plants (liquorice and Alisma orientalis; [45]). All specimens contained mercury, with the highest content of $23 \mu \mathrm{g} / \mathrm{g}$ (!) found in one commercial product. The product's exact composition was not specified, but results like these definitely indicate serious risks for the consumers.

\section{Fingerprinting \\ $\nabla$}

An analytical approach that is especially employed on TCM is most likely to be fingerprinting. It does not denote the use of particular techniques but encompasses a unique style of data evaluation. Due to the complex composition of herbal drugs in general, and of multi-herb formulations in particular, this approach has several advantages for the quality evaluation of TCM preparations compared to conventional analysis. The latter usually enables the quantitative determination of individual compounds (marker compounds) that are either unique for a particular species or responsible for biological activity. But they fail to provide a complete picture of a herbal product composed of a multitude of constituents, whether they are active or not, specific or common. Fingerprinting tries to overcome this weakness by establishing chromatographic, electrophoretic or spectroscopic patterns. Based on the "similarity" and "integrity" of these patterns in comparison to a reference (e.g., clinically proven product), authentication and identification of herbal medicines is possible even when the amount of characteristic constituents is not the same $[46,47]$. It is not required to resolve all compounds in the extract, but if certain compounds are separated, quantitative determinations are also possible.

Since 2004 the Chinese State Food and Drug Administration (SFDA) requires all injections made from TCM raw materials to be standardized using chromatographic fingerprints [47]. But, as can be seen in Table 1, the vast number of respective reports is not limited to injectibles alone or to chromatographic methods [48-61]. Most of the reports describe the application on a single plant and not multi-herb preparations. In many cases HPLC is utilized for fingerprinting, e.g., on Angelica dahurica [48], Atractylis chinensis [50], Ganoderma lucidum [54] or ginkgo [55]. Other techniques such as gas chromatography (Rehmannia glutinosa, [57]), capillary electrophoresis (Salvia miltiorrhiza, [58]) and IR spectroscopy (Angelica sinensi, [49]) were successfully used as well. Concerning multi-plant preparations, the standardization of "Danning tablets" [60] and "Longdan-Xiegan decoction" [61] 
by HPLC fingerprints are good examples. The latter comprises 10 medicinal plants including Gentianae radix, Scutellarae radix, Plantaginis semen and Alismathis rhizoma. For establishing chromatographic patterns 2-dimensional HPLC was used. The first separation was achieved on silica material modified with human serum albumin (therefore the authors used the term biochromatography for their study). The second separation was performed on a silica monolithic column. More than 100 compounds could be separated, with 19 of them being identified based on their mass spectra. Compared to routine approaches the authors considered their method advantageous because of "enhanced peak capacity, good sensitivity and powerful resolution".

Different strategies for evaluating results can be found in the literature, but the majority of reports were done either using principal component analysis (PCA) or the commercially available software "Similarity evaluation system for chromatographic fingerprint of TCM" (Chinese Pharmacopoeia Committee, 2004). Based on peak areas, retention times, and after assigning marker peaks, a mean chromatogram is generated. This is compared with the actually obtained results, and based on the calculated correlation coefficient sample consistency can be determined [48]. Metabolomic profiling can be seen as another form of fingerprint analysis, aiming to identify the complete set of metabolites in tissues or cells without bias. Currently NMR- or MS-based approaches are mainly used for this purpose whereby the sample (e.g., plant extract) is directly determined without any prior separation. Once again, the profiles obtained are observed for compound specific signals or the total fingerprint is compared [62]. Besides recent reviews $[63,64]$, another contribution to this issue of Planta Medica further elaborates on these particular techniques. Hence, only a few successful applications on plants with TCM relevance are mentioned here. They include fresh ginseng roots [65], Angelica acutiloba [66], different Ephedra species [67] and Artemisia annua [68].

\section{Other Approaches \\ $\nabla$}

Alternative methods for controlling the quality of TCM not described in the previous sections are the biological approaches. A few interesting reports can be found in current literature, such as the quantitative determination of saikosaponins in several $\mathrm{Bu}$ pleurum species ("Chai-Hu" in Chinese) on polyether sulphone membranes with monoclonal antibodies [69]; the analysis of glycyrrhizin and glycyrrhizic acid in diverse preparations by ELISA (enzyme-linked immunosorbent assay [70]); or using gene expression for this purpose [71]. In the last example human hepatocellular carcinoma cells (HepG2) were used as a "dynamic detector". They were incubated with a complex TCM decoction, and the expression on selected marker genes was monitored by quantitative PCR analysis. Compared to HPLC results this biological method was shown to be equally capable of distinguishing between different batches of the investigated decoction.

\section{Conclusions}

\section{$\nabla$}

According to EMEA (European Medicines Agency) guidelines on the "Quality of herbal medicinal products" for each herbal substance and preparation a comprehensive specification is required. Agreement with these specifications has to be confirmed by control tests, allowing the qualitative and quantitative deter- mination of active substances or specific markers [72]. As shown in this review, within the last few years a multitude of new techniques and applications has been developed and reported, so that tools for resolving this quality problem seem to be available. Whether consumers will actually benefit from this improved situation or not is now up to the willingness of regulatory affairs and manufacturers.

\section{References}

1 Xinhua W, Changguo W, Xiaoyun M. Basic theory of traditional Chinese medicine, 1st edition. Shanghai: Publishing House of Shanghai University of Traditional Chinese Medicine; 2002: 1-10

2 Bensky D, Clavey S, Stöger E. Chinese herbal medicine - materia medica, 3rd edition. Seattle: Eastland Press; 2004: 13-31

3 Hempen $\mathrm{CH}$, Fischer T. Leitfaden Chinesische Phytotherapie, 2nd edition. München: Urban \& Fischer; 2007: 2-19

4 Li SL, Lin G, Fu PP, Chan CL, Li M, Jiang ZH, Zhao ZZ. Identification of five hepatotoxic pyrrolizidine alkaloids in a commonly used traditional Chinese medicinal herb, Herba Senecionis scandentis (Qianliguang). Rapid Commun Mass Spectrom 2008; 22: 591-602

5 Wu KM, Farrelly JG, Upton R, Chen J. Complexities of herbal nomenclature system in traditional Chinese medicine (TCM): lessons learned from the misuse of Aristolochia-related species and the importance of the pharmaceutical name during botanical drug product development. Phytomedicine 2007; 14: 273-279

$6 \mathrm{Li}$ W, Chen Z, Liao Y, Liu H. Separation methods for toxic components in traditional Chinese medicines. Anal Sci 2005; 21: 1019-1029

7 Liu S, Yi LZ, Liang, YZ. Traditional Chinese medicine and separation science. J Sep Sci 2008; 31: 2113-2137

8 Liu Z. Preparation of botanical samples for biomedical research. Endocrine Metab Immune Disord Drug Targ 2008; 8: 112-121

9 Deng C, Liu N, Gao M, Zhang X. Recent developments in sample preparation techniques for chromatography analysis of traditional Chinese medicines. J Chromatogr A 2007; 1153: 90-96

$10 \mathrm{Li}$ Y, Wang Y, Qu H, Cheng Y. Simultaneous determination of seven bioactive compounds in Chinese medicine Qi-Shen-Yi-Qi dropping pill by LC-UV and LC-ELSD. Chromatographia 2008; 67: 293-297

11 Su J, Fu P, Shen Y, Zhang C, Liang M, Lui R, Li H, Zhang W. Simultaneous analysis of flavonoids from Hypericum japonicum Thunb. ex Murray (Hypericaceae) by HPLC-DAD-ESI/MS. J Pharm Biomed Anal 2008; 46: 342-348

12 Zheng $X$, Shi P, Cheng Y, Qu $H$. Rapid analysis of a Chinese herbal prescription by liquid chromatography-time-of-flight tandem mass spectrometry. J Chromatogr A 2008; 1206: 140-146

13 Chan EC, Yap SL, Lau AJ, Leow PC, Toh TF, Koh HL. Ultra-performance liquid chromatography/time-of-flight mass spectrometry based metabolomics of raw and steamed Panax notoginseng. Rapid Commun Mass Spectrom 2008; 21: 519-528

14 Dan M, Su M, Gao X, Zhao T, Zhao A, Xie G, Qiu Y, Zhou M, Liu Z, Jia W. Metobolite profiling of Panax notoginseng using UPLC-ESI-MS. Phytochemistry 2008; 69: 2237-2244

15 Xie G, Plumb R, Su M, Xu Z, Zhao A, Qiu M, Long X, Liu Z, Jia W. Ultraperformance LC/TOF MS analysis of medicinal Panax herbs for metabolomic research. J Sep Sci 2008; 31: 1015-1026

16 Chen XJ, Ji H, Zhang QW, Tu PF, Wang YT, Guo BL, Li SP. A rapid method for simultaneous determination of 15 flavonoids in Epimedium using pressurized liquid extraction and ultra-performance liquid chromatography. J Pharm Biomed Anal 2008; 46: 226-235

17 Jin Y, Zhang X, Shi H, Xiao YS, Ke YX, Xue YX, Zhang FF, Liang XM. Characterisation of C-glycosyl quinochalcones in Carthamus tinctorius L. by ultra-performance liquid chromatography coupled with quadrupoletime-of-flight mass spectrometry. Rapid Commun Mass Spectrom 2008; 22: 1275-1287

18 Maruyama T, Kamakura H, Miyai M, Komatsu K, Kawasaki T, Fujita M, Shimada H, Yamamoto Y, Shibata T, Goda Y. Authentication of traditional medicinal plant Eleutherococcus senticosus by DNA and chemical analysis. Planta Med 2008; 74: 787-789

19 Hemström P, Irgum K. Hydrophilic interaction chromatography. J Sep Sci 2006; 29: 1784-1821

20 Wang Y, Lu X, Xu G. Simultaneous separation of hydrophilic and hydrophobic compounds using an online HILIC-RPLC system with two detectors. J Sep Sci 2008; 31: 1564-1572 
21 Davis JM, Giddings JC. Statistical theory of component overlap in multicomponent chromatograms. Anal Chem 1983; 55: 418-424

$22 \mathrm{Hu} L$, Chen X, Kong L, Su X, Ye M, Zou H. Improved performance of comprehensive two-dimensional HPLC separation of traditional Chinese medicines using a silica monolithic column and normalization of peak heights. J Chromatogr A 2005; 1092: 191-198

23 Liu Y, Xue X, Guo Z, Xu Q, Zhang F, Liang X. Novel two-dimensional liquid chromatography/hydrophilic interaction chromatography, an excellent orthogonal system for practical analysis. J Chromatogr A 2008; 1208: $133-140$

24 Ma S, Chen L, Luo G, Ren K, Wu J, Wang Y. Off-line comprehensive twodimensional high-performance liquid chromatography system with size exclusion column and reverse phase column for separation of complex traditional Chinese medicine Qingkailing injection. J Chromatogr A 2006; 1127: 207-213

25 Deng C, Ji J, Wang X, Zhang X. Development of pressurized hot water extraction followed by headspace solid-phase microextraction and gas chromatography-mass spectrometry for determination of ligustilides in Ligusticum chuanxiong and Angelica sinensis. J Sep Sci 2005; 28: 1237-1243

26 Shen S, Sha Y, Deng C, Zhang X, Fu C, Chen J. Quality assessment of Flos Chrysanthemi indici from different growing areas in China by solidphase microextraction-gas chromatography-mass spectrometry. J Chromatogr A 2004; 1047: 281-287

27 Dong L, Wang J, Deng C, Shen X. Gas chromatography-mass spectrometry following pressurized hot water extraction and solid-phase microextraction for quantification of eucalyptol, camphor, and borneol in Chrysanthemum flowers. J Sep Sci 2007; 30: 80-89

28 Li N, Mao Y, Deng C, Zhang X. Separation and identification of volatile constituents in Artemisia argyi flowers by GC-MS with SPME and steam distillation. J Chromatogr Sci 2008; 46: 401-405

29 Deng C, Yao N, Wang B, Zhang X. Development of microwave assisted extraction followed by headspace dingle-droplet microextraction for fast determination of paeonol in traditional Chinese medicines. J Chromatogr A 2006; 1103: 15-21

30 Dong L, Deng C, Wang B, Shen X. Fast determination of Z-ligustilide in plasma by gas-chromatography/mass spectrometry following headspace single-droplet extraction. J Sep Sci 2007; 30: 1318-1325

31 Ito J, Bowman RL. Countercurrent chromatography: liquid-liquid chromatography without solid support. Science 1970; 167: 281-283

32 Ito $Y$. Golden rules and pitfalls in selecting optimum conditions for high-speed counter-current chromatography. J Chromatogr A 2005; 1065: 145-168

33 Wang X, Liu J, Thang T, Ito Y. Rapid and simple method for quality control of raw materials of herbs by HSCCC. J Liquid Chromatogr Res Technol 2007; 30: 2585-2592

34 Ganzera $M$. Quality control of herbal medicines by capillary electrophoresis: potential, requirements and applications. Electrophoresis 2008; 29: 3489-3503

35 Chen Y, Fan G, Chen B, Xie Y, Wu H, Wu Y, Yan C, Wang Y. Separation and quantitative analysis of coumarin compounds from Angelica dahurica (Fisch. ExHoffm.) Benth. et Hook. f. by pressurized capillary electrochromatography. J Pharm Biomed Anal 2006; 41: 105-116

36 Yang F, Li S, Li P, Wang Y. Optimization of CEC for simultaneous determination of eleven nucleosides and nucleobases in Cordyceps using central composite design. Electrophoresis 2007; 28: 1681-1688

37 Chen X, Ji H, Wang Y, Li S. Simultaneous determination of seven flavonoids inEpimedium using pressurized liquid extraction and capillary electrochromatography. J Sep Sci 2008; 31: 881-887

38 Eeltink S, Svec F. Recent advances in the control of morphology and surface chemistry of porous polymer-based monolithic stationary phases and their application in CEC. Electrophoresis 2007; 28: 131-147

39 Lü H, Wang J, Wang X, Lin X, Wu X, Xie Z. Rapid separation and determination of structurally related anthraquinones in rhubarb by pressurized liquid chromatography. J Pharm Biomed Anal 2007; 43: 352-357

40 Zhang L, Zhang J, Wang H, Zhang L, Zhang W, Zhang Y. Analysis of leaves of Adinandra nitida by capillary electrochromatography on monolithic columns with stepwise gradient elution. J Sep Sci 2005; 28: 774-779

41 Dong J, Ou J, Dong X, Wu R, Ye M, Zou H. Preparation and evaluation of rigid porous polyacrylamide-based strong cation-exchange monolithic columns for capillary electrochromatography. J Sep Sci 2007; 30: 2986-2992

42 Lin X, Wang J, Li L, Wang X, Lü H, Xie Z. Separation and determination of five major opium alkaloids with mixed mode of hydrophilic/cationic exchange monolith by pressurized capillary electrochromatography. J Sep Sci 2007; 30: 3011-3017

43 Huang $H, Y u H, X u H$, Ying $Y$. Near infrared spectroscopy for on/in-line monitoring of quality in foods and beverages: a review. J Food Eng 2008; 87: 303-313

44 Wu YW, Sun SQ Zhou Q Leung HW. Fourier transform mid-infrared (MIR) and near-infrared (NIR) spectroscopy for the rapid quality assessment of Chinese medicine preparation Honghua oil. J Pharm Biomed Anal 2008; 46: 498-504

45 Huang RJ, Zhuang ZX, Tai Y, Huang RF, Wang XR, Lee F. Direct analysis of mercury in traditional Chinese medicines using thermolysis coupled with on-line atomic absorption spectrometry. Talanta 2006; 68: 728734

46 Jia Y, Tan X, Li Q Cheng W, Chen X, Bi K. Quality control of TCMs using a chromatographic fingerprint. Asian J Trad Med 2006; 1: 94-100

47 Liang YZ, Xie P, Chan K. Quality control of herbal medicines. J Chromatogr B 2004; 812: 53-70

48 Kang J, Zhou L, Sun J, Ham J, Guo DA. Chromatographic fingerprint analysis and characterisation of furanocoumarins in Angelica dahurica by

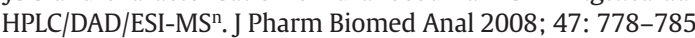

49 Liu H, Sun S, Lv G, Liang X. Discrimination of extracted lipophilic constituents of Angelica with multi-steps infrared macro-fingerprint method. Vib Spec 2006; 40: 202-208

50 Ni Y, Peng Y, Kokot S. Fingerprinting of complex mixtures with the use of high performance liquid chromatography, inductively coupled plasma atomic absorptionemission spectroscopy and chemometrics. Anal Chim Acta 2008; 616: 19-27

$51 \mathrm{Li} \mathrm{Y,} \mathrm{Hu} \mathrm{Z,} \mathrm{He} \mathrm{L.} \mathrm{An} \mathrm{approach} \mathrm{to} \mathrm{develop} \mathrm{binary} \mathrm{chromatographic} \mathrm{finger-}$ prints of the total alkaloids from Caulophyllum robustum by high performance liquid chromatography/diode array detector and gas chromatography/mass spectrometry. J Pharm Biomed Anal 2007; 43: $1667-1672$

52 Chen J, Wang F, Liu J, Lee F, Wang X, Yang H. Analysis in Coptis chinensis Franch by accelerated solvent extraction combined with ultra performance liquid chromatographic analysis with photodiode array and tandem mass spectrometry detections. Anal Chim Acta 2008; 613: 184-195

53 Pei LK, Sun SQ Guo BL, Huang WH, Xiao PG. Fast quality control of Herba Epimedii by using Fourier transform infrared spectroscopy. Spectrochim Acta A 2008; 70: 258-264

54 Chen Y, Yan Y, Xie MY, Nie SP, Liu W, Gong XF, Wang YX. Development of a chromatographic fingerprint for the chloroform extracts of Ganoderma lucidum by HPLC and LC-MS. J Pharm Biomed Anal 2008; 47: 469477

55 Sun G, Liu J. Qualitative and quantitative assessment of the HPLC fingerprints of Ginkgo biloba extract by the involution similarity method. Anal Sci 2007; 23: 955-958

56 Xie G, Zhao A, Li P, Li L, Jia W. Fingerprint analysis of Rhizoma chuanxiong by pressurized capillary electrochromatography and high performance liquid chromatography. Biomed Chromatogr 2007; 21: 867875

57 Chang WT, Thissen U, Ehlert KA, Koek M, Jellema R, Hakemeier T, van der Greef J, Wang M. Effects of growth conditions and processing on Rehmannia glutinosa using fingerprint strategy. Planta Med 2006; 72: 458-467

58 Gu M, Zhang S, Su Z, Chen Y, Ouyang F. Fingerprinting of Salvia miltiorrhiza Bunge by non-aqueous capillary electrophoresis compared with high-speed counter-current chromatography. J Chromatogr A 2004; 1057: $133-140$

$59 \mathrm{Gu}$ M, Su Z, Ouyang F. Fingerprinting of Salvia miltiorrhiza Bunge by thin-layer chromatography scan compared with high-speed countercurrent chromatography. J Liquid Chromatogr Res Technol 2006; 29: 1503-1514

60 Liang M, Zhang J, Liu R, Yan S, Zhang W, Lin M. Chemical profile of Danning tablets by LC-DAD coupled with ESI-MS. Chromatographia 2007; 66: $107-110$

61 Wang Y, Kong L, Hu L, Lei X, Yang L, Chou G, Zuo H, Wang C, Bligh SWA, Wang $Z$. Biological fingerprinting analysis of the traditional Chinese prescription Longdan Xiegan decoction by on/off-line comprehensive two-dimensional biochromatography. J Chromatogr B 2007; 860: 185-194

62 Krishnan P, Kruger NJ, Ratcliffe RG. Metabolite fingerprinting and profiling in plants using NMR. J Exp Bot 2005; 56: 255-265

63 Holmes E, Tang $H$, Wang $Y$, Seger $C$. The assessment of plant metabolite profiles by NMR-based methodologies. Planta Med 2006; 72: 771-785 
64 Seger C, Sturm S. Analytical aspects of plant metabolite profiling platforms: current standings and future aims. J Proteome Res 2007; 6: 480-497

65 Shin YS, Bang KH, In DS, Kim OT, Hyun DY, Ahn IO, Ku BC, Kim SW, Seong $N S$, Cha SW, Lee D, Choi HK. Fingerprinting analysis of fresh ginseng roots of different ages using ${ }^{1} \mathrm{H}-\mathrm{NMR}$ spectroscopy and principal components analysis. Arch Pharm Res 2007; 30: 1625-1628

66 Tarachiwin L, Katoh A, Ute K, Fukusaki E. Quality evaluation of Angelica acutiloba Kitagawa roots by $1 \mathrm{H}$ NMR-based metabolic fingerprinting. J Pharm Biomed Anal 2008; 48: 42-48

67 Kim HK, Choi YH, Erkelens C, Lefeber A, Verpoorte R. Metabolic fingerprinting of Ephedra species using 1H-NMR spectroscopy and principal component analysis. Chem Pharm Bull 2005; 53: 105-109

$68 \mathrm{Ma}$ C, Wang $H, \mathrm{Lu} X, \mathrm{Xu} \mathrm{G}$, Liu B. Metabolic fingerprinting investigation of Artemisia annua L. in different stages of development by gas chromatography and gas chromatography-mass spectrometry. J Chromatogr A 2008; 1186: 412-419
69 Morinaga O, Zhu S, Tanaka H, Shoyama Y. Visual detection of saikosaponins by on-membrane immunoassay and estimation of traditional Chinese medicines containing Bupleuri Radix. Biochem Biophys Res Commun 2006; 346: 687-692

70 Xu J, Tanaka H, ShoyanaY. One-step immunochromatographic separation and ELISA quantification of glycyrrhizin from traditional Chinese medicines. J Chromatogr B 2007; 850: 53-58

71 Rong J, Tilton R, Shen J, Ng KM, Liu C, Tam P, Lau ASY, Cheng YC. Genomewide biological response fingerprinting (BioReF) of the Chinese botanical formulation ISF-1 enables the selection of multiple marker genes as a potential metric for quality control. J Ethnopharmacol 2007; 113: 3544

72 Guideline on the quality of herbal medicinal products. Available at http://www.emea.europa.eu/pdfs/human/qwp/281900en.pdf. Accessed March 10, 2009 